\title{
Digital Control of PWM Inverter Using Ultrahigh-Speed Network for Feedback Signals with Communication Disturbance Observer Based on Rocket I/O Protocol
}

\author{
Ryo Saito* Non-member, Kazuo Tsuchida** Non-member \\ Tomoki Yokoyama* Member
}

(Manuscript received Aug. 16, 2014, revised Feb. 17, 2015)

\begin{abstract}
A new network communication system for a power electronics controller that transmits the feedback signal from the sensors using an FPGA-based hardware controller was proposed. Based on the RocketI/O network controller, a customized Rocket I/O protocol was implemented to achieve high-speed feedback communication between two FPGA controllers. One-megahertz variable sampling quasi-multirate deadbeat control was applied for the PWM inverter control. The 1-MHz sampled data were transmitted using the Rocket I/O protocol and proper operation of the feedback control was experimentally verified. To compensate for the network delay of the feedback signal, a communication disturbance observer was combined with the network feedback system. The advantage of the proposed method was verified.
\end{abstract}

Keywords: network feedback, rocket I/O, FPGA, PWM inverter, digital control

\section{Introduction}

To construct the power electronics equipments, various sensors are necessary for the measurement of the state variables and these signals have to feedback to control the system. These signals are connected by metal wire from the sensors to the control board. In the case of rather large capability power electronics system, multi-cell or multi-unit system is generally applied and the number of feedback signals becomes huge, so many wire cables are necessary to build up the system. This result in the prevention of the reduction of the manufacturing cost and the complexity of the system.

On the other hand, progress of network technology makes it possible to realize the real time high-speed communication system to apply to the power electronics system. It is expected that the data feedback of power electronics will shift to the digital data communication using network from the conventional transfer technique of analog signal in the future. In order to realize the transform of the feedback signal using network communication in practical use, it is necessary to consider the trade-off of the merit (reduction of the wire) and the demerit (restriction of transmission speed, the quality and safety of communication pathway).

Authors reported the network feedback system based on the ethernet protocol using FPGA based hardware controller $^{(1)}$. In that case, the overhead of the ethernet protocol was rather large, it was hard to construct real time

\footnotetext{
${ }^{*}$ Department of Robotics and Mechatronics, Graduate school of Science and Technology for Future Life, Tokyo Denki University

5, Asahicho, Senju, Adachi-ku, Tokyo 120-8551, Japan

${ }^{* *}$ Fuji Electric Co., Ltd.

1, Fuji-cho, Hino-city, Tokyo 191-8502, Japan
}

digital feedback system with the network feedback. But recently, the ultrahigh-speed real time network protocol was announced and implemented to the basic feature of FPGA chip. Rocket $\mathrm{I} / \mathrm{O}^{(2)}$ is one of these network protocol and the communication speed becomes up to $3.125 \mathrm{G} \mathrm{bit} / \mathrm{sec}$ which is suitable to apply to power electronics system. In this research, the network communication method for power electronics is proposed and verified using FPGA based hardware controller. The ultrahigh-speed network communication system for power electronics based on Rocket I/O protocol was designed and implemented to FPGA chip ${ }^{(3)}$. And the power electronics controller was also implemented to the same FPGA chip. Using FPGA, all the protocol processing is implemented in the hardware, the network transmission speed becomes maximum, the protocol processing is treated by the high-speed control circuit. $1 \mathrm{MHz}$ variable sampling quasi multi-rate deadbeat control ${ }^{(4)}$ was used as the digital control method to evaluate the network feedback control for the PWM inverter. This method uses $1 \mathrm{MHz}$ sampled data while the carrier interval and is suitable for the evaluation the potential of Rocket I/O protocol.

Through the experiments, the accurate operations of the data communication were verified, and the network transfer delay of the data is observed around $0.136 \mu$ seconds. Communication disturbance observer (CDOB) was introduced to compensate the network delay of the feedback signal. Network disturbance was defined to simulate the network conditions.

If the network delay is larger than around 10 us, the $\mathrm{CDOB}$ is effective to suppress the affection of the network delay. But in the case that the network delay is smaller than that range, the effectiveness of $\mathrm{CDOB}$ is not so remarkable and is considered that it is installed as the failsafe of the network 
disturbance. Through the simulations and the experiments, the proper operation of the network feedback system using Rocket I/O protocol was confirmed and verified, also the effectiveness of the communication disturbance observer was verified in the case that the network delay is larger than 10 us.

\section{Digital Control with Network Feedback}

2.1 System Configuration Figure 1 shows the system configuration of the proposed method. The system is configured with PWM inverter and two FPGA controllers equipped with the Rocket I/O network driver. Board 1 treats the $\mathrm{A} / \mathrm{D}$ interface and transfer the converted digital data into the packet then pass to the Rocket I/O transceiver. Board 2 treats the packet receiving and take out the feedback data to the inverter control block, then the digital control and communication disturbance observer were applied to the system. In each board, all the calculations were implemented in one FPGA chip, and all the functions for the network communication were processed in that FPGA chip.

2.2 Rocket I/O Transceiver In this research, all the control system is built in the FPGA board. The FPGA board is equipped with the Rocket $\mathrm{I} / \mathrm{O}$ transceiver, and the Rocket I/O transceiver is connected to the FPGA directly. The Virtex-5 FPGA XC5 V-LX110T-PCIEXP is used as a FPGA based hardware controller. The proposed system communicates all the feedback data through the Rocket I/O transceiver.

In the Virtex 5 chip, the Rocket I/O transceiver is implemented in the chip itself. PHY structure is used to communicate with the FPGA through the physical layer, so the control block can access to Rocket I/O network directly in the FPGA chip.

\subsubsection{Transmission Function and Receiving Function}

The transmission function and receiving function were applied to the transmitter of the Rocket I/O transceiver. These functions were configured by the PCS layer and the PMA layer. The data coding, decoding and the control of the signal integrity were performed in the PCS layer. Parallel to serial conversion of the data is carried out in the PMA layer. Figure 2 shows a block diagram of a transmitter.

2.2.2 Communication Speed and Construction of the FPGA Module The theoretical value of the transmitting processing time was calculated from the VHDL code of the proposed system as follows.

- Transmitting processing time : $238[\mathrm{nsec}]$

- Receiving processing time : 319 [nsec]

The total maximum time of the theoretical value for the transmitting process and the receiving process becomes

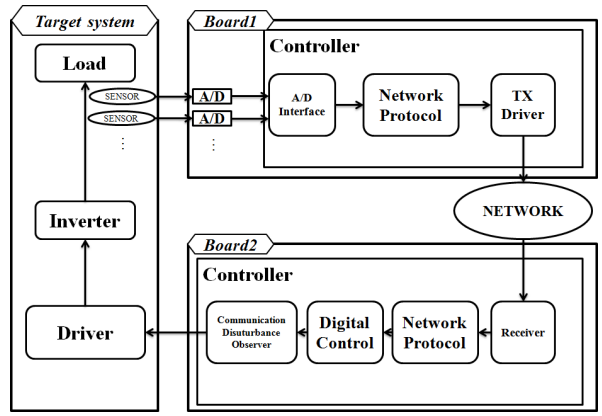

Fig. 1. Configuration of proposed system $557.6[\mathrm{nsec}]$. So the practical network delay of the Rocket $\mathrm{I} / \mathrm{O}$ is assumed within $557.6[\mathrm{nsec}]$.

2.3 $1 \mathrm{MHz}$ Variable Sampling Quasi Multi-rate Deadbeat Control (1MVSQMDB) ${ }^{(4)} \quad$ To evaluate the performance of the network feedback system, $1 \mathrm{MHz}$ variable sampling quasi multi-rate deadbeat control was applied as the control law. This method uses $1 \mathrm{MHz}$ sampled data as the feedback signal, which is suitable for the Rocket I/O feedback system. Figure 3 shows the system configuration of the proposed method. The state equation of the continuous time model of the PWM inverter becomes as Eq. (1).

$$
\begin{gathered}
\dot{x}(t)=A_{c} x(t)+B_{c} u(t) \ldots \ldots \ldots \ldots \ldots \ldots \ldots \ldots \ldots \ldots \ldots \\
, \text { where } \quad x(t)=\left[\begin{array}{cc}
v_{o}(t) & \dot{v}_{o}(t)
\end{array}\right]^{T} \\
, A_{C}=\left[\begin{array}{cc}
0 & 1 \\
-\frac{1}{L C} & -\frac{1}{R C}
\end{array}\right], \quad B_{C}=\left[\begin{array}{c}
0 \\
\frac{1}{L C}
\end{array}\right]
\end{gathered}
$$

To measure the $\dot{v}_{o}, I_{c}$ is measured in this system, and $I_{c}$ can be converted to $\dot{v}_{o}$. To reflect the $1 \mathrm{MHz}$ sampled data immediately to the PWM pulse width, three case of PWM pulse pattern is considered for the modeling as shown in Fig. 4. In Fig. 4(a) and (b), the pulse is outputted in the center of the carrier period, and in Fig. 4(c), the pulse is outputted from the starting point of the carrier period. In the case of Fig. 4(a), the sampled data of $k$ 's instant is used, the discrete time model becomes as follows,

$$
\begin{gathered}
x[k+1]=A_{d} x[k]+B_{d} u_{c}[k] \cdots \ldots \ldots \\
, \text { where } A_{d}=e^{A_{c} T_{u}}, B_{d}=e^{\frac{A_{c} T_{u}}{2}} B_{c} E .
\end{gathered}
$$

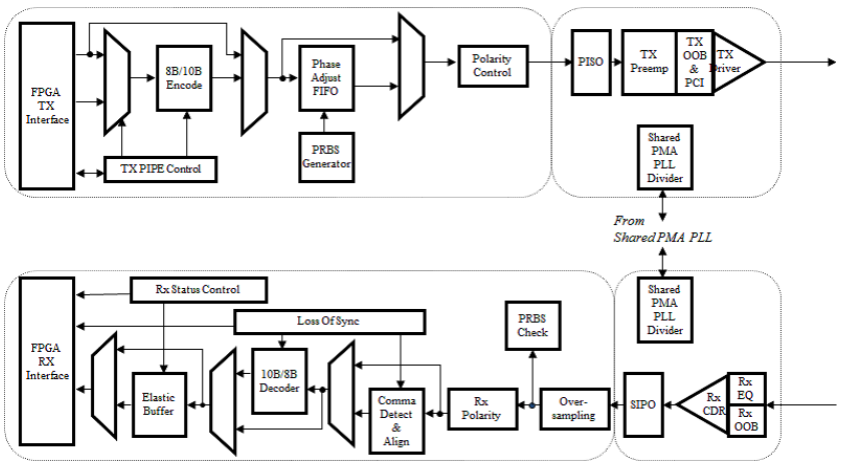

Fig. 2. Transmission block and receiving block

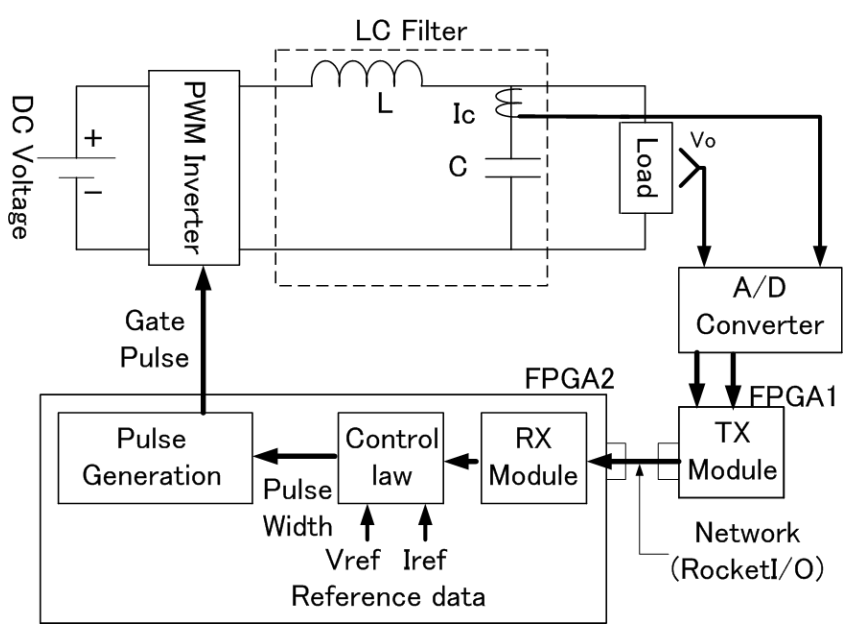

Fig. 3. Single phase PWM inverter 


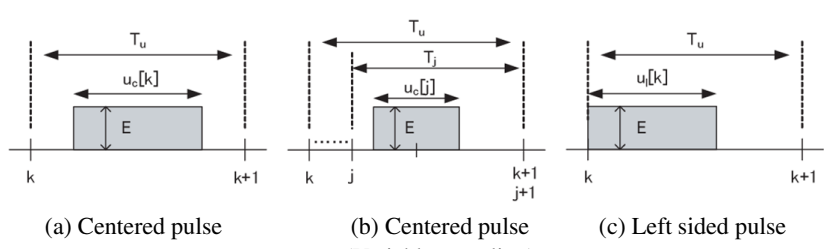

Fig. 4. Discrete time model

In the case of Fig.4(c), the discrete time model becomes as follows,

$$
\begin{gathered}
x[k+1]=A_{d l} x[k]+B_{d l} u_{l}[k] \cdots \cdots \cdots \\
, \text { where } A_{d l}=e^{A_{c} T_{u}}, B_{d l}=e^{A_{c} T_{u}} B_{c} E .
\end{gathered}
$$

In the case of Fig. 4(b), from the starting point of the carrier, $1 \mathrm{MHz}$ data sampling is carried out for the state variables in every $j$ 's instant. In this case, the discrete time model becomes Eq. (4). So the sampling interval $T_{j}$ is varied in every sampling instant, the matrix $A_{d j}$ in Eq. (4) should be recalculated in every $j$ 's sampling instant.

$$
\begin{gathered}
x[j+1]=A_{d j} x[j]+B_{d} u[j] \\
, \text { where } A_{d j}=e^{A_{c} T_{j}} .
\end{gathered}
$$

From Eq. (2), the discrete time model of the next carrier period becomes Eq. (5). Using Eq. (4) and Eq. (5), multi-rate model of this system can be derived as Eq. $(6)^{(5)}$.

$$
\begin{gathered}
x[k+2]=A_{d} x[k+1]+B_{d} u[k+1] \ldots \ldots \ldots \ldots \\
\left.x[k+2]=A_{m j} x[j]+B_{m}\left[u_{c}[j], u_{c}[k+1]\right]\right]^{T} \ldots \ldots \\
, \text { where } A_{m j}:=A_{d} A_{d j} B_{m}:=\left[A_{d} B_{d}, B_{d}\right] .
\end{gathered}
$$

As the same way, using Eq. (3), the multi-rate model of Eq. (3) becomes Eq. (7).

$$
x[k+2]=A_{d l}^{2} x[k]+\left[A_{d l} B_{d l}, B_{d l}\right]\left[u_{l}[k], u_{l}[k+1]\right]^{T}
$$

Equation (7) can be rewritten to Eq. (8) while the carrier interval.

$$
\begin{gathered}
x[i+1]=A_{m l} x[i]+B_{m l} u_{l}[i] \ldots \ldots \ldots \\
, \text { where } \quad u_{l}[i]=\left[u_{l}[k], u_{l}[k+1]\right]^{T} \\
, A_{m l}:=A_{d l}^{2}, B_{m l}:=\left[A_{d l} B_{d l}, B_{d l}\right] .
\end{gathered}
$$

To improve the robustness for the parameter variations, quasi multi-rate deadbeat control method is combined to $1 \mathrm{MHz}$ variable sampling deadbeat control method. In the multi-rate control method, two pulse width are derived in Eq. (6) and Eq. (8) for the 2nd order system. Using these two equations, multi-rate pulse width Eq. (9) and Eq. (10) can be derived. Equation (6) can be rewritten to Eq. (9) while the carrier interval of Fig. 5(a), and Eq. (8) can be rewritten to Eq. (10) while the carrier interval of Fig. 5(b). To avoid the sampling frequency becomes half of the carrier frequency, two pulse width are combined to one pulse as shown in Fig. 5. While the sampling duration is mode I, the centered pulse method in Fig. 5(a) is applied, and in the case of mode III, the left sided pulse method in Fig. 5(b) is applied respectively. Figure 5(a) adds the pulse width $u_{c}[j]$ and $u_{c}[k+1]$ of Eq. (9), and Fig. 5(b) adds the pulse width $u_{l}[n]$ and $u_{l}[n+1]$ of Eq. (10).

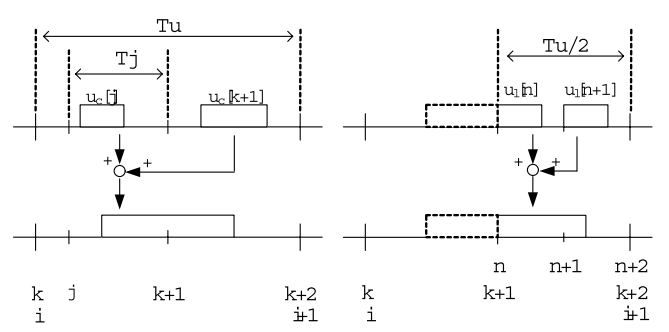

(a) Centered pulse

(b) Left sided pulse

Fig. 5. Pulse pattern of quasi multi-rate deadbeat control

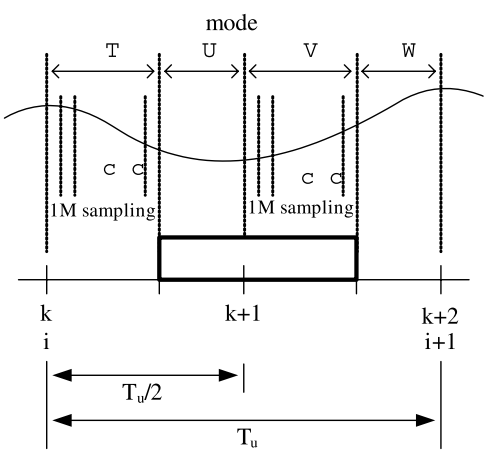

Fig. 6. Sampling procedure of variable sampling deadbeat control method

$$
\begin{aligned}
& {\left[u_{c}[j], u_{c}[k+1]\right]^{T}=B_{m}^{-1} x[k+2]-B_{m}^{-1} A_{m j} x[j] \cdots} \\
& u_{l}[k+1]=B_{m l}^{-1} x[k+2]-B_{m l}^{-1} A_{m l} x[k+1] \cdots \cdots
\end{aligned}
$$

Figure 6 shows the timing chart of 1MVSQMRDB. One carrier interval is divided into four mode. In the mode I, using Eq. (9), the pulse width is calculated using every $1 \mathrm{MHz}$ sampled data, and wait for the instant that the present instant is included to the calculated pulse width, then the pulse is turned on. In the mode II, $1 \mathrm{MHz}$ sampled data is not used until the center of the carried interval. In the mode III, using Eq. (10), the pulse width is calculated using every $1 \mathrm{MHz}$ sampled data, waiting for the instant that the present instant is not included to the calculated pulse width, then the pulse is turned off. In the mode IV, waiting for the next carrier interval. Switching timing is controlled based on $125 \mathrm{MHz}$ system clock of the FPGA to achieve the precise PWM control. As the result, while the carrier interval, $1 \mathrm{MHz}$ sampled data can be reflected to the output pulse width to improve the tracking accuracy to the reference voltage. This control method recalculates the pulse width during the carrier interval, the PWM pulse generation circuit should be respond to the recalculated pulse width immediately. So the FPGA based hardware controller is applied to realize 1MVSQMRDB. All the calculation including the conversion time of $\mathrm{A} / \mathrm{D}$ converter can be finished around $985.3 \mathrm{~ns}$ which is not including the network delay time.

2.4 Implementation of Rocket I/O System In the proposed system, Rocket I/O was used to realize ultrahighspeed feedback data transmission. 13 bit A/D converter is applied in this system, 16 bit data band is prepared to construct data packet. Figure 7 shows the RTL (Register Transfer Level) diagram of the communication module and $A / D$ module. VHDL module configuration of the board 1 (A/D interface and packet generation) was indicated in Fig. 8. Rocket $\mathrm{I} / \mathrm{O}$ module treats the packet generation procedure 


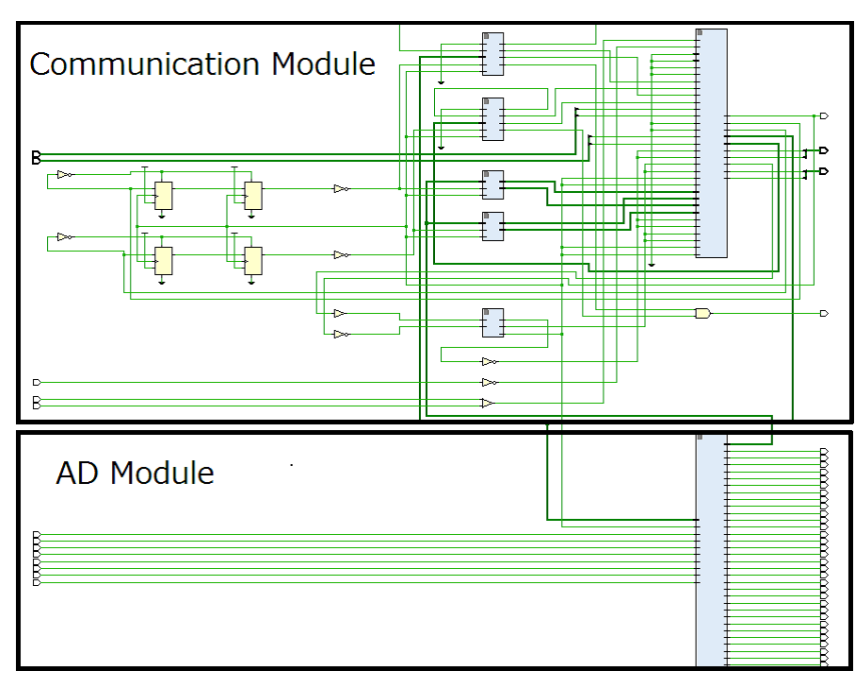

Fig. 7. RTL diagram of rocket $\mathrm{I} / \mathrm{O}$ and $\mathrm{A} / \mathrm{D}$ control module

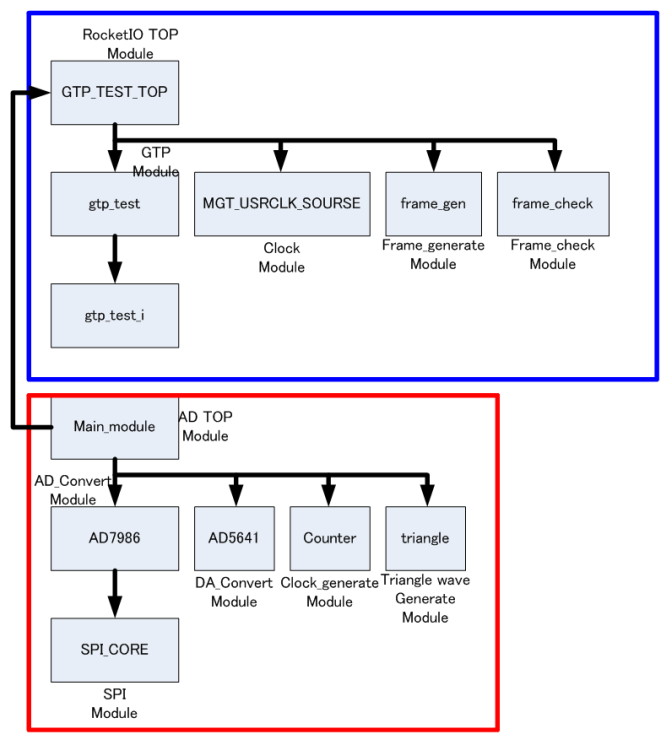

Fig. 8. VHDL module configuration of rocket I/O

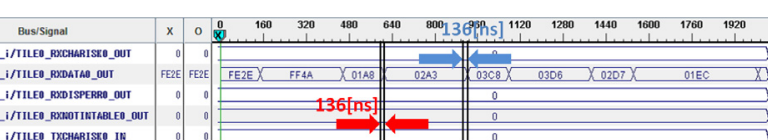

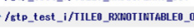

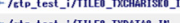

Fig. 9. Transmitting and receiving data

and A/D module treats the interface between FPGA and A/D converter.

2.5 Experimental Result of Rocket I/O Figure 9 shows the experimental packet data of Rocket I/O using the proposed system. Data transmission function between the board 1 and the board 2 was confirmed. The total time of the experimental value for the transmitting process and the receiving process becomes $136[\mathrm{nsec}]$ as indicated in Fig. 9.

2.6 Communication Disturbance Observer (CDOB)

In the case that the network is used for the feedback signal, the network delay is exist in the feedback line. Communication disturbance observer (CDOB) was proposed to compensate the network delay in which the communication delay was treated as the disturbance ${ }^{(6)-(8)}$. In this paper, CDOB was adopted to the network feedback to compensate the network

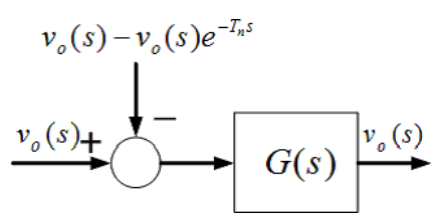

Fig. 10. Network disturbance

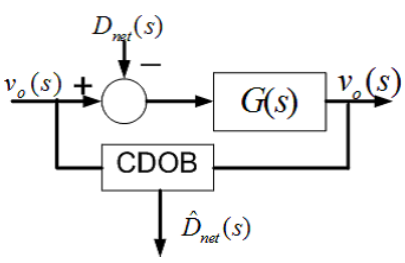

Fig. 11. Communication disturbance observer

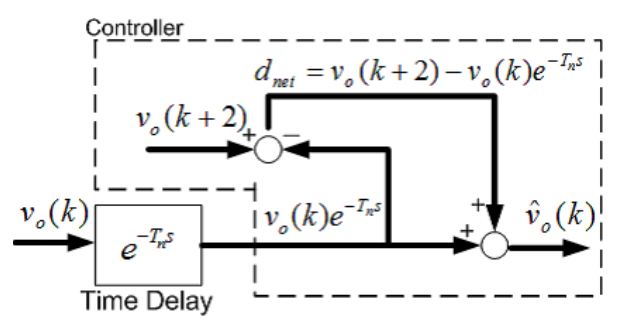

Fig. 12. Communication disturbance observer for $v_{o}(k)$

delay.

Figure 10 indicates the concept of network disturbance (ND) and Fig. 11 shows the control block diagram of the network disturbance. First-order CDOB was considered to assume that the ND is the polynomial of time domain $t$. ND was defined as follows.

$$
\begin{aligned}
& d_{n e t}(t)=v_{o}(t)-v_{o}\left(t-T_{n}\right) \\
& \dot{d}_{n e t}(t)=0 \ldots \ldots \ldots \ldots \ldots
\end{aligned}
$$

$T_{n}$ is the delay time of the network. ND was treated as the vector in Eq. (13).

$$
\boldsymbol{d}_{\text {net }}(t)=\left[\begin{array}{c}
d_{n e t}(t) \\
\dot{d}_{\text {net }}(t)
\end{array}\right]
$$

Then the ND can be estimated as follows in the first-order CDOB. $g_{n e t}$ is the cut-off frequency gain of the low pass filter for the first-order CDOB.

$$
\hat{D}_{n e t}(s)=\frac{g_{\text {net }}}{s+g_{\text {net }}} D_{n e t}(s)
$$

ND can be described in the discrete time domain as follows.

$$
\begin{aligned}
& d_{n e t}(t)=v_{o}(k+2)-v_{o}(k) e^{-T_{1} s} . \\
& \dot{d}_{n e t}(t)=\dot{v}_{o}(k+2)-\dot{v}_{o}(k) e^{-T_{1} s} .
\end{aligned}
$$

From these equations, $\hat{v}_{o}$ and $\hat{v_{o}}$ can be derived as follows.

$$
\begin{aligned}
& \hat{v}_{o}(k+2)=\hat{d}_{n e t}(t)+v_{o}(k) e^{-T_{1} s} . \\
& \hat{\dot{v}}_{o}(k+2)=\hat{\dot{d}}_{n e t}(t)+\dot{v}_{o}(k) e^{-T_{1} s} .
\end{aligned}
$$

Figure 12 shows the block diagram of CDOB for $v_{o}$ and Fig. 13 shows the block diagram of CDOB for $\dot{v}_{o}$. Equation (17) and Eq. (18) can be used as the compensation value for the network delay. Then Eq. (10), Eq. (17) and Eq. (14) can be combined, the $\mathrm{CDOB}$ of the proposed system can be 


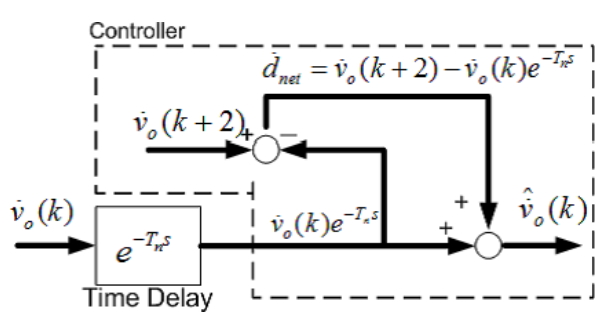

Fig. 13. Communication disturbance observer for $\dot{v}_{o}(k)$

Table 1. System parameters

\begin{tabular}{|l|l|}
\hline Output voltage frequency $[\mathrm{Hz}]$ & 50 \\
\hline Output voltage amplitude $\left[V_{r m s}\right]$ & 20 \\
\hline Input dc voltage $[\mathrm{V}]$ & 40 \\
\hline Sampling Time $[\mathrm{ms}]$ & 0.5 \\
\hline Simulation Time $[\mathrm{ms}]$ & 0.2 \\
\hline Carrier Frequency $[\mathrm{kHz}]$ & 20 \\
\hline L & $2.0[\mathrm{mH}]$ \\
\hline
\end{tabular}

\begin{tabular}{|l|l|}
\hline $\mathrm{C}$ & $30.39[\mu \mathrm{F}]$ \\
\hline$g_{\text {net }}$ & 1.0 \\
\hline Load Conditions \\
Resistive load $\quad 50[\Omega]$ \\
Rectifier load R $50[\Omega]$ \\
\\
\multicolumn{2}{|c|}{$0.6[\mathrm{mH}]$} \\
& $\mathrm{C} 660[\mu \mathrm{F}]$ \\
\hline
\end{tabular}

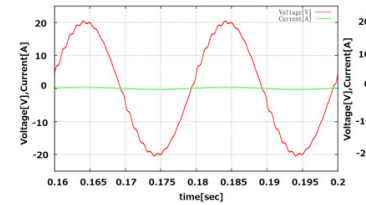

(a) with $\mathrm{CDOB}$

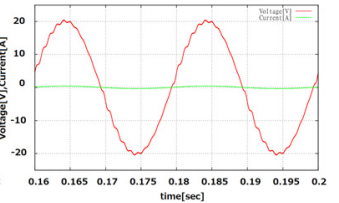

(b) without $\mathrm{CDOB}$
Fig. 14. Voltage and current waveforms for resistive load with or without $\mathrm{CDOB}$

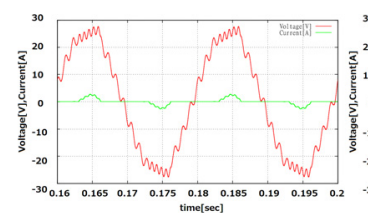

(a) with $\mathrm{CDOB}$

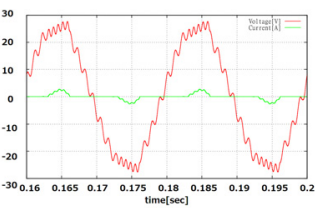

(b) without $\mathrm{CDOB}$
Fig. 15. Voltage and current waveforms for rectifier load with or without $\mathrm{CDOB}$

described as follows.

$$
u_{l}[k+1]=B_{m l}^{-1}\left[\begin{array}{c}
\hat{v}_{o}[k+2] \\
\hat{v}_{o}[k+2]
\end{array}\right]-B_{m l}^{-1} A_{m l} x[k+1]
$$

\section{Simulation}

Simulations were carried out to evaluate the effectiveness of 1MVSQMDB and the CDOB with the network delay conditions, the following conditions were used to evaluate the output characteristics.

THD and the steady state error of the output voltage are used as the criteria for the evaluation. Table 1 shows the system parameters for the simulations.

Figure 14 shows the voltage and current waveforms for resistive load with or without CDOB, and Fig. 15 shows the voltage and current waveforms for rectifier load with or without CDOB. In both cases, the delay time of the network was settled to $557.6 \mathrm{~ns}$, which is the theoretical maximum delay time of Rocket I/O. The network speed of Rocket I/O is very fast, not so much difference can be observed. Figure 16 and Fig. 17 show the THD trend of the output voltage and the steady state error of the output voltage when the network delay was varied from 0 us to 200 us respectively. As shown in Fig. 16, the affection of the network delay was suppressed

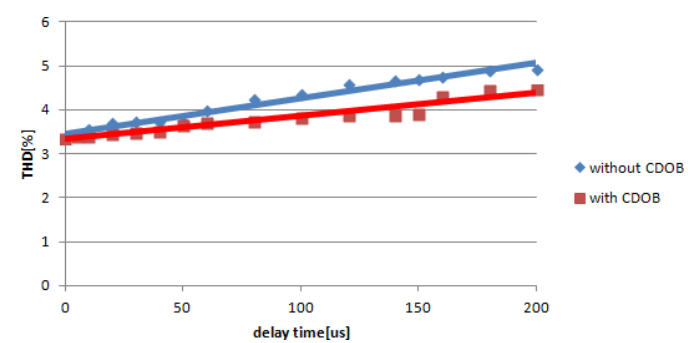

Fig. 16. Network delay time vs. THD of $v_{o}$

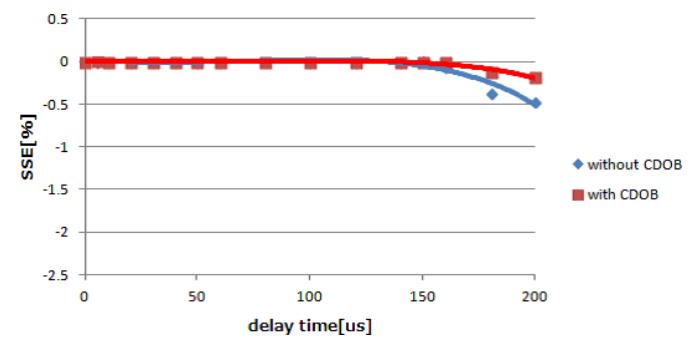

Fig. 17. Network delay time vs. steady state error of $v_{o}$

Table 2. System parameters

\begin{tabular}{|c|c|}
\hline Output voltage frequency [Hz] & 50 \\
\hline Output voltage amplitude $\left[V_{r m s}\right]$ & 28 \\
\hline Input dc voltage [V] & 40 \\
\hline L & $2.0[\mathrm{mH}]$ \\
\hline $\mathrm{C}$ & $30.39[\mu \mathrm{F}]$ \\
\hline Load Conditions \\
Resistive load $50.0[\Omega]$ \\
Rectifier load R $50[\Omega]$ \\
L $0.6[\mathrm{mH}]$ \\
C $660[\mu \mathrm{F}]$ \\
\hline
\end{tabular}

with CDOB. If the network delay is larger than around 10 us, the CDOB is effective to suppress the affection of the network delay. But in the case that the network delay is smaller than around $10 \mathrm{us}$, the difference with or without CDOB is very small. As shown in Fig. 17, the affection of the network delay was suppressed with CDOB when the delay time is larger than 130 us. From these results, CDOB is not so effective when the network delay is rather small but it can be considered that it is installed as the failsafe of the network disturbance.

\section{Experimental Result}

Experiments were carried out for the PWM inverter using FPGA based hardware controller and the network feedback system with Rocket I/O protocol. Practical network delay of Rocket I/O was 136 [ nsec] as described in Sect. 2.5. Comparisons were carried out in the case that the CDOB was applied to the network feedback or not.

CDOB is applied to both of voltage feedback and current feedback.

Figure 18 shows the voltage and current waveforms for resistive load with $\mathrm{CDOB}$ and Fig. 19 shows that of without CDOB. Figure 20 shows the voltage and current waveforms for rectifier load with CDOB and Fig. 21 shows that of without CDOB. These results were summarized in Table 3.

In these cases, the proper operation of the network feedback system using Rocket I/O protocol was confirmed. As discussed in Sect. 3, the network delay time of the Rocket $\mathrm{I} / \mathrm{O}$ protocol is smaller than $557.6 \mathrm{~ns}$, so the effectiveness of 


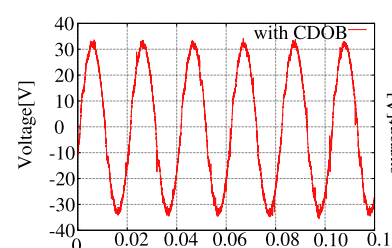

time[sec]

(a) Voltage

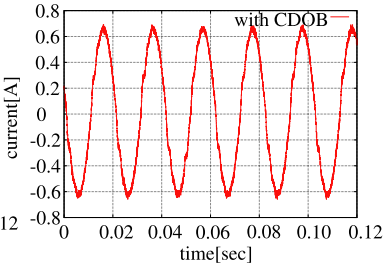

(b) Current
Fig. 18. Voltage and current waveforms for resistive load with $\mathrm{CDOB}$

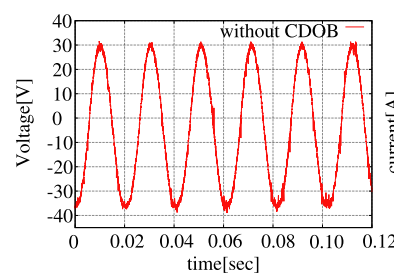

(a) Voltage

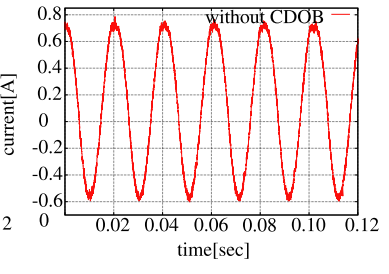

(b) Current
Fig. 19. Voltage and current waveforms for resistive load without $\mathrm{CDOB}$

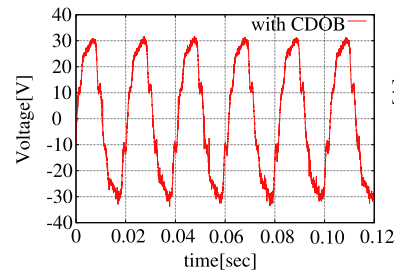

(a) Voltage

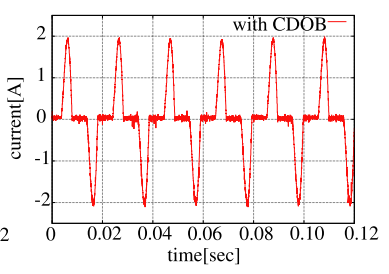

(b) Current
Fig. 20. Voltage and current waveforms for rectifier load with $\mathrm{CDOB}$

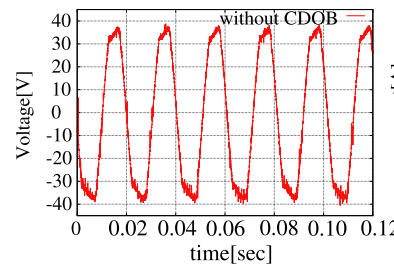

(a) Voltage

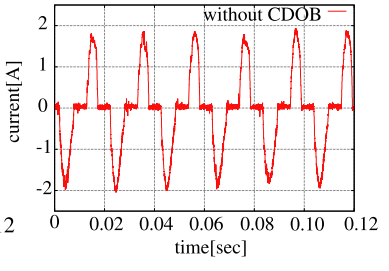

(b) Current
Fig. 21. Voltage and current waveforms for rectifier load without $\mathrm{CDOB}$

Table 3. Experimental result

\begin{tabular}{|c|c|c|c|}
\hline & RMS[V] & SSE[EE & THD[EE \\
\hline resistive load without CDOB & 23.7 & 1.67 & 6.86 \\
\hline rectifier load without CDOB & 24.7 & 2.25 & 12.6 \\
\hline resistive load with CDOB & 23.3 & 1.63 & 5.21 \\
\hline rectifier load with CDOB & 22.0 & 1.20 & 13.5 \\
\hline
\end{tabular}

CDOB is not so remarkable. As shown in Table 3, the THD characteristics and the steady state error are not so much different in the case of with or without CDOB. It can be observed that the Rocket I/O protocol is fast enough to use for the network feedback of the inverter control. The basic operation of the inverter control using network feedback system was verified.

\section{Conclusion}

An ultra fast network feedback system using Rocket I/O protocol was implemented to the digital control system of PWM inverter using FPGA based hardware controller. $1 \mathrm{MHz}$ variable sampling quasi multi-rate deadbeat control was applied and the communication disturbance observer was combined to compensate the network delay of the feedback signal. The proper operation of $1 \mathrm{MHz}$ sampling and network feedback was experimentally confirmed and the network delay becomes 136 [ $\mathrm{nsec}$ ], an ultra fast network feedback system was verified. Also the effectiveness of the communication disturbance observer for the network delay was confirmed in the case that the network delay is larger than $10 \mu \mathrm{s}$. The proposed method is useful for the future power electronics system with network based digital control system.

\section{References}

(1) S. Kojima, T. Ishioka, and T. Yokoyama: "A study of Communication System for Power Electronics Controller using FPGA based Hardware Controller", IPEC (2010)

( 2 ) "XILINX Corporation" http://japan.xilinx.com/

( 3 ) K. Tsuchida and T. Yokoyama: "Verification of high-speed network communication system using FPGA", JIASC (2012)

( 4 ) H. Uchida and T. Yokoyama: "1 MHz Variable Multi Sampling Digital Control of Single Phase PWM Inverter using FPGA based Hardware Controller", ECCE (2012)

( 5 ) A. Kawamra, H. Fujimoto, and T. Yokoyama: "Survey on the real time digital feedback control of PWM inverter and the extension to multi-rate sampling and FPGA based inverter control", in Proc. Annual Conference of the IEEE Industrial Electronics Society (IECON'07), pp.2044-2051, Taipei (2007)

( 6 ) K. Natori, R. Oboe, and K. Ohnishi: "Stability Analysis and Practical Design Procedure of Time Delayed Control Systems with Communication Disturbance Observer", IEEE Trans. on Industrial Informatics, Vol.4, No.3, pp.185-197 (2008)

( 7 ) K. Natori, R. Kubo, T. Shimono, and K. Ohnishi: "Time-Delay Compensation by Communication Disturbance Observers of Different Orders in Bilateral Teleoperation Systems", IEEJ, pp.353-362 (2009)

( 8 ) M. Kitamura: "Expansion of Control Algorithm for Reconfigurable Robots", Master's Thesis 2011, Mie University Graduate School of Regional Innovation Studies (2011)

Ryo Saito (Non-member) was born in Kanagawa Prefecture, Japan in

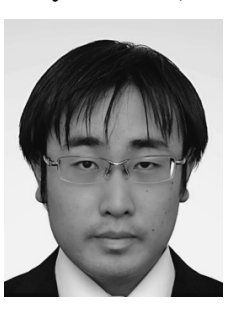
1990. He received the B.S. and M.S. degrees in Robot Mechatronics from Tokyo Denki University, Tokyo, Japan, in 2013 and 2015 respectively.

Kazuo Tsuchida (Non-member) was born in Chiba Prefecture, Japan

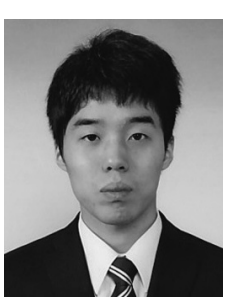
in 1989. He received the B.S. and M.S. degrees in Robot Mechatronics from Tokyo Denki University, Tokyo, Japan, in 2012 and 2014 respectively. In 2014, he joined Fuji Electric Corporation Japan. His research interests are in the areas of advanced control of power electronics.

Tomoki Yokoyama (Member) was born in Hiroshima Prefecture,

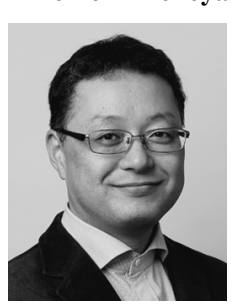
Japan in 1965. He received the B.S., M.S. and Ph.D. degrees in Electrical Engineering from Yokohama National University, Kanagawa, Japan, in 1988 and 1994 respectively. In 1994, he joined Toshiba Corporation Japan. In 1998, he joined Tokyo Denki University. He is currently an associate professor. His research interests are in the areas of advanced control of power electronics. Prof. Yokoyama is a member of the Institute of Electrical Engineers of Japan. 